\title{
Schistosomiasis among obstetric fistula patients in Lilongwe, Malawi
}

\section{Laura B. Drew ${ }^{1}$, Jennifer H. Tang ${ }^{2,3}$, Alison Norris ${ }^{4}$, Patricia Carr Reese $^{5}$, Mwawi Mwale ${ }^{6}$, Ronald Mataya ${ }^{7,8}$, Jeffrey P. Wilkinson ${ }^{9,10}$}

1. University of Maryland, School of Public Health

2. UNC Project-Malawi

3. UNC Department of Obstetrics and Gynaecology

4. The Ohio State University, College of Public Health

5. The George Washington University, School of Medicine and Health Sciences

6. Lilongwe District Health Office

7. Loma Linda University School of Public Health

8. Malawi College of Medicine, Department of Obstetrics and Gynaecology

9. Freedom from Fistula Care Centre

10. Baylor College of Medicine

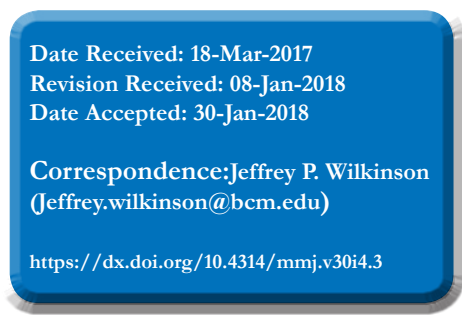

\section{Background}

Abstract

Schistosoma baematobium infection has been documented as an uncommon cause of vesicovaginal fistula (VVF) and can result in impaired wound healing of urogenital tissues. For these reasons, it could potentially be linked to an increased rate of obstetric fistula among women who experience obstructed labor and/or in a higher failure rate of fistula repair. Therefore, the primary objective of our study was to determine the prevalence of $S$. haematobium infection among women undergoing obstetric VVF repair in Lilongwe, Malawi. Our secondary objectives were to assess if $S$. haematobium infection could be a risk factor for obstetric fistula development or unsuccessful VVF repair in our patient population.

Methods

From July to October 2013, we conducted S. haematobium testing via urine microscopy on 96 patients undergoing obstetric VVF repair surgery at the Fistula Care Centre in Lilongwe, Malawi.

Results

The prevalence of $S$. haematobium infection among women undergoing obstetric VVF repair was $2 \%$ ( $=2$ ). Both women with $S$. haematobium had successful VVF repairs.

Conclusions

Although S. haematobium has the potential to be a risk factor for obstetric VVF formation or unsuccessful VVF repair, it was uncommon among the women in our clinic with obstetric VVF.

Key words: fistula, vesicovaginal fistula, schistosomiasis, schistosoma, schistosoma haematobium

\section{Introduction}

An estimated 230 million individuals worldwide have schistosomiasis ${ }^{1}$, and more than $90 \%$ of these infections occur in Africa ${ }^{2-4}$. Schistosoma haematobium is the cause of urogenital schistosomiasis infections; of the estimated 43 million adults in Sub-Saharan Africa who have symptoms of $S$. haematobium infection, more than $16 \%$ have major bladder wall pathology $y^{5,6}$. Nearly $75 \%$ of girls and women with chronic $S$. baematobium infection develop female genital schistosomiasis (FGS), a condition in which schistosoma egg deposition throughout the urogenital tract causes granulomas, sandy patches, and rubbery papules on the uterus, cervix, and lower genital tract ${ }^{2,7-9}$. One estimate suggests that 19 million girls and women currently infected with S. haematobium globally will develop FGS in the next 10 years ${ }^{2,10}$.

In Malawi, chronic infections with schistosomiasis are common and contribute to significant morbidity ${ }^{11}$. The most recent national estimate of the prevalence of schistosomiasis in Malawi was estimated to be between $40 \%$ and $50 \%$ of the population ${ }^{12}$. However, the National Schistosomiasis Control Programme used surveys conducted among highrisk populations prior to 1991 to calculate this national estimate. A more recent survey in 2002 incorporating highand low-risk populations estimated the overall prevalence of S. haematobium among primary school children in Malawi to be $6.9 \%$. The prevalence varied substantially by region, ranging from $2.0-23.2 \%$ for $S$. haematobium ${ }^{13}$.

Obstetric fistula $(\mathrm{OF})$ is a urogynecologic condition that is typically a consequence of prolonged, obstructed labor. During obstructed labor, the soft tissues of the pelvis undergo necrosis from lack of blood supply. This necrosis results in an abnormal opening between the vagina and the bladder and/or rectum with subsequent incontinence of urine and/or feces ${ }^{14}$. Most of cases in the developing world are vesicovaginal fistulas $(\mathrm{VVF})^{15}$, which involve an abnormal opening between the bladder and the vagina. In Malawi, the estimated prevalence of VVF is 1.6 in 1000 women $^{16}$.

A few case studies have addressed the relationship between schistosomiasis and VVF; however, none specifically detail an association between schistosomiasis and $\mathrm{OF}^{5,17,18}$. Given that the presence of $S$. haematobium in the bladder could lead to inflammation, fibrosis, rupture, ulceration, and potentially fistula ${ }^{5,19,20}$, it could also be associated with formation of OF or with delays in healing from VVF repair surgery. Despite the biologic plausibility of $S$. haematobium contributing to OF, a 1970 study by Bland and Gelfand did not find an association between urinary schistosomiasis infection and $\mathrm{OF}^{19}$. However, this cross-sectional study found a significant association between schistosomiasis infection and failure 
to heal after fistula repair. Treatment or pretesting for schistosoma ova in the urine of patients with VVF and/or obstetric fistula could be an important part of pre-operative care in areas endemic for $S$. haematobium ${ }^{5,17}$.

Therefore, the primary objective of our study was to determine the prevalence of $S$. baematobium infection among women undergoing obstetric VVF repair at a fistula repair center in Lilongwe, Malawi. Our secondary objectives were to assess if $S$. haematobium infection could be a risk factor for OF development or unsuccessful VVF repair in our patient population.

\section{Methods}

From July to October 2013, urinary schistosomiasis testing was conducted on 96 patients who had OF repair surgery at the Fistula Care Centre at Bwaila Hospital in Lilongwe, Malawi. Most of the patients at this clinic are from Central Malawi, including the lakeshore region. Patients who had VVF due to non-obstetric causes were not included in this study.

Women who consented to participate in our study had $50 \mathrm{~mL}$ of urine collected from their urinary catheter bags within 24 hours of undergoing surgery. The urine specimens were immediately delivered to the UNC Project laboratory at Bwaila Hospital for urine microscopy to detect the presence of $S$. haematobium ova. Upon arrival in the lab, the urine specimen was centrifuged, and then a few drops of the sediment were placed on a slide and reviewed under the microscope. Microscopy results were documented in the patients' charts, and women who had schistosomiasis were treated with praziquantel $(40 \mathrm{mg} / \mathrm{kg})$. While pre-operative testing for schistosoma ova in the urine of women with VVF has been suggested ${ }^{5,17}$, we found this suggestion to be impractical as our patients had constant leaking of small quantities of urine, which was not sufficient for collection in a urine specimen cup or urinary catheter.

Nurses and clinicians, fluent in Chichewa, documented the following information for all study participants: demographics, clinical history, and physical exam information, as well as preoperative, operative, and postoperative findings. HIV testing was done preoperatively and dye tests were done postoperatively (at least 14 days after surgery) to identify whether the fistula repair was successful.

Women were asked to provide written informed consent in Chichewa. Participants under the age of 17 completed both a Pediatric Assent and Parental Consent Form. The UNC School of Medicine Biomedical Institutional Review Board and the Institutional Review Board of the National Health Sciences Research Committee of Malawi approved the consent form and all study materials.

Microscopy results and other study data were entered into the REDCap database for the Fistula Care Centre by trained research associates ${ }^{21}$. Stata 12.1 software was used for statistical analyses (StataCorp LP, College Station, TX). We chose a convenience sample of up to 100 women who had obstetric VVF repair between July and October 2013.

\section{Results}

The mean age of the 96 women included in the study was 37.7 years (range 17-77) (Table 1). Most women were from districts in Central Malawi (78\%). All but two women were from rural areas, and most were farmers $(84 \%)$. Most women were Christian (90\%).
Table 1: Baseline characteristics of study participants ${ }^{\mathrm{a}, \mathrm{b}}$

\begin{tabular}{|c|c|c|c|}
\hline Patient characteristics, $n=96$ & $\begin{array}{c}\text { Schistosomiasis } \\
\text { negative, } \mathrm{n}=94\end{array}$ & $\begin{array}{c}\text { Schistosomiasis } \\
\text { positive, } n=2\end{array}$ & $\begin{array}{l}\text { Total } \\
\mathrm{n}(\%)\end{array}$ \\
\hline \multicolumn{4}{|l|}{ Age (mean $=37.7$ years) } \\
\hline$<20$ & $3(3 \%)$ & $0(0 \%)$ & $3(3 \%)$ \\
\hline $20-29$ & $27(29 \%)$ & $1(50 \%)$ & $28(29 \%)$ \\
\hline $30-39$ & $23(24 \%)$ & $1(50 \%)$ & $24(25 \%)$ \\
\hline $40-49$ & $21(22 \%)$ & $0(0 \%)$ & $21(22 \%)$ \\
\hline $50-59$ & $10(11 \%)$ & $0(0 \%)$ & $10(10 \%)$ \\
\hline $60-69$ & $7(7 \%)$ & $0(0 \%)$ & $7(7 \%)$ \\
\hline$>70$ & $2(2 \%)$ & $0(0 \%)$ & $2(2 \%)$ \\
\hline Unknown & $1(1 \%)$ & $0(0 \%)$ & $1(1 \%)$ \\
\hline \multicolumn{4}{|l|}{ Religion } \\
\hline Christian & $85(90 \%)$ & $1(50 \%)$ & $86(90 \%)$ \\
\hline Muslim & $7(7 \%)$ & $1(50 \%)$ & $8(8 \%)$ \\
\hline Traditional & $1(1 \%)$ & $0(0 \%)$ & $1(1 \%)$ \\
\hline None & $1(1 \%)$ & $0(0 \%)$ & $1(1 \%)$ \\
\hline \multicolumn{4}{|l|}{ Type of Residence } \\
\hline Urban & $2(2 \%)$ & $0(0 \%)$ & $2(2 \%)$ \\
\hline Rural & $92(98 \%)$ & $2(100 \%)$ & $94(98 \%)$ \\
\hline \multicolumn{4}{|l|}{ District of Residence } \\
\hline \multicolumn{4}{|l|}{ Northern Malawi } \\
\hline Chitipa & $1(1 \%)$ & $0(0 \%)$ & $1(1 \%)$ \\
\hline Karonga & $1(1 \%)$ & $0(0 \%)$ & $1(1 \%)$ \\
\hline Mzimba & $6(6 \%)$ & $0(0 \%)$ & $6(6 \%)$ \\
\hline \multicolumn{4}{|l|}{ Central Malawi } \\
\hline Dedza & $13(14 \%)$ & $0(0 \%)$ & $13(14 \%)$ \\
\hline Dowa & $9(10 \%)$ & $0(0 \%)$ & $9(9 \%)$ \\
\hline Kasungu & $9(10 \%)$ & $0(0 \%)$ & $9(9 \%)$ \\
\hline Lilongwe & $26(28 \%)$ & $0(0 \%)$ & $26(27 \%)$ \\
\hline Mchinji & $2(2 \%)$ & $0(0 \%)$ & $2(2 \%)$ \\
\hline Nkhotakota & $6(6 \%)$ & $0(0 \%)$ & $6(6 \%)$ \\
\hline Ntcheu & $5(5 \%)$ & $0(0 \%)$ & $5(5 \%)$ \\
\hline Ntchisi & $1(1 \%)$ & $0(0 \%)$ & $1(1 \%)$ \\
\hline Salima & $4(4 \%)$ & $0(0 \%)$ & $4(4 \%)$ \\
\hline \multicolumn{4}{|l|}{ Southern Malawi } \\
\hline Balaka & $1(1 \%)$ & $0(0 \%)$ & $1(1 \%)$ \\
\hline Machinga & $3(3 \%)$ & $0(0 \%)$ & $3(3 \%)$ \\
\hline Mangochi & $1(1 \%)$ & $2(100 \%)$ & $3(3 \%)$ \\
\hline Mulanje & $1(1 \%)$ & $0(0 \%)$ & $1(1 \%)$ \\
\hline Mozambique $^{c}$ & $5(5 \%)$ & $0(0 \%)$ & $5(5 \%)$ \\
\hline \multicolumn{4}{|l|}{ Education completed } \\
\hline None & $40(43 \%)$ & $2(100 \%)$ & $42(44 \%)$ \\
\hline Standard 1-Standard 7 & $50(53 \%)$ & $0(0 \%)$ & $50(52 \%)$ \\
\hline Secondary or more & $2(2 \%)$ & $0(0 \%)$ & $2(2 \%)$ \\
\hline Standard 8 & $1(1 \%)$ & $0(0 \%)$ & $1(1 \%)$ \\
\hline Unknown & $1(1 \%)$ & $0(0 \%)$ & $1(1 \%)$ \\
\hline \multicolumn{4}{|l|}{ Occupation } \\
\hline Farmer & $79(84 \%)$ & $2(100 \%)$ & $81(84 \%)$ \\
\hline Student & $1(1 \%)$ & $0(0 \%)$ & $1(1 \%)$ \\
\hline Unemployed & $1(1 \%)$ & $0(0 \%)$ & $1(1 \%)$ \\
\hline Housewife/caretaker & $3(3 \%)$ & $0(0 \%)$ & $3(3 \%)$ \\
\hline Small business owner & $8(9 \%)$ & $0(0 \%)$ & $8(8 \%)$ \\
\hline Other & $1(1 \%)$ & $0(0 \%)$ & $1(1 \%)$ \\
\hline Unknown & $1(1 \%)$ & $0(0 \%)$ & $1(1 \%)$ \\
\hline
\end{tabular}

${ }^{a}$ Collected at first visit to Fistula Care Centre

${ }^{\mathrm{b}}$ Percentages do not add up to $100 \%$ due to rounding

c Women who live along the border of Mozambique and Malawi often receive health care in Malawi because our hospitals and health centers are actually closer to their homes. 
Table 2: Clinical data from study participants ${ }^{\mathrm{a}}$

\begin{tabular}{lccc}
\hline & $\begin{array}{c}\text { Schistosomiasis } \\
\text { negative, } n=94\end{array}$ & $\begin{array}{c}\text { Schistosomiasis } \\
\text { positive, } n=2\end{array}$ & Total \\
Clinical notes, $n=96$ & & $n(\%)$ \\
\hline
\end{tabular}

Surgery number at

Fistula Care Centre

$79(84 \%)$

$2(100 \%)$

$81(84 \%)$

$1^{\text {st }}$

$11(12 \%)$

$0(0 \%)$

$11(12 \%)$

$2^{\text {nd }}$

$4(4 \%)$

$0(0 \%)$

$4(4 \%)$

$3^{\text {rd }}$

HIV status

$$
\text { Positive }
$$

$6(6 \%)$

$0(0 \%)$

$6(6 \%)$

Negative

$88(94 \%)$

$2(100 \%)$

$90(94 \%)$

Previously sought

treatment for leakage

$64(68 \%)$

$2(100 \%)$

$66(69 \%)$

Yes

$30(32 \%)$

$0(0 \%)$

$30(31 \%)$

No

Previous operation to fix

leakage

$22(23 \%)$

$0(0 \%)$

$22(23 \%)$

Yes

$72(77 \%)$

$2(100 \%)$

$74(77 \%)$

No

${ }^{a}$ Percentages do not add up to $100 \%$ due to rounding or missing data

The majority of women had previously sought treatment for their leakage (69\%); however, only a third of those who sought treatment had prior surgeries to repair their fistulas. Most women had little (52\%) or no education (44\%).

The prevalence of $S$. haematobium infection among women with obstetric fistula was $2 \%(n=2)$ (Table 2). The two patients who tested positive for S. haematobium were both from lakeshore areas in Mangochi district. One woman was 35 years old and one was 20 years old. Both women resided in rural areas, were farmers, and had not completed any education. Although both women had previously sought treatment for urinary leakage, neither woman had a previous operation to repair her fistula.

The postoperative dye test results for both women with schistosomiasis were negative, indicating that their repairs were successful. All six women who had positive postoperative dye test results (indicating failed fistula repair) were schistosomiasis negative.

\section{Discussion}

S. haematobium infection was rare among women undergoing surgical repair of obstetric VVF at our Fistula Care Centre in Lilongwe, Malawi. In addition, none of the women who had a failed VVF repair were positive for $S$. baematobium. Therefore, our data do not support the theory that $S$. haematobium infection plays a role in obstetric VVF development or unsuccessful VVF repair in Malawi.

Praziquantel is often given empirically to people in communities in Malawi with high rates of schistosomiasis to treat and control schistosomiasis ${ }^{22}$. The success of this program may have contributed to the low prevalence of $S$. haematobium in our study ${ }^{13}$. The two women who had schistosomiasis were from lakeshore areas with the highest rates of $S$. haematobium infection in Malawi ${ }^{13}$. Although S. haematobium is considered endemic in Malawi, our OF patient population had a prevalence similar to the lower range of prevalence in the general population of Malawi ${ }^{13}$. Because most of our patients came from areas with a low schistosomiasis prevalence, routine screening for $S$. haematobium in our patient population would not be cost-effective given that each test costs USD $\$ 4$. However, screening could be performed and cost-effectively for those fistula patients coming to the hospital from areas with a high prevalence of $S$. haematobium. Further studies to clarify the most cost-effective screening approach are warranted.

A study by Bland and Gelfand found the incidence of $S$. haematobium infection of the bladder to be no higher in obstetric VVF patients than in a control series ${ }^{19}$. In that study, $40(67 \%)$ of 60 women in the OF group and $36(60 \%)$ of 60 in the control group (women admitted to the gynecologic ward without $\mathrm{OF}$ ) had $S$. haematobium, which was not a statistically significant difference. However, among the 60 women undergoing OF repair, they did find a statistically significant difference in fistula repair failure. Of the 40 women with $S$. haematobium, $25(62.5 \%)$ had an unsuccessful fistula repair, whereas among the 20 women without S. haematobium, only 6 $(30 \%)$ had an unsuccessful fistula repair.

Our study has a few important differences from the Bland and Gelfand study ${ }^{19}$. First, our study did not include control subjects, although we did find that the prevalence of $S$. haematobium among our OF population was similar to the prevalence in the general population of Malawi. Secondly, the Bland and Gelfand study was done from 1966 to 1968 in an area that is now part of Zimbabwe and had a much higher prevalence for $S$. baematobium. The high prevalence in that study may be due to a lack of empiric treatment programs for schistosomiasis at that time and also due to the fact that the authors tested for S. haematobium via urine microscopy, rectal snips, and bladder biopsy, whereas we only used urine microscopy. Finally, our study differed from theirs in postoperative care and evaluation for residual fistula. In the Bland and Gelfand study, the urinary catheter was usually left in place for 18 days after surgery (catheter removal at 14-21 days is the standard ${ }^{23}$, and assessment for residual fistula was done by evaluation under anesthesia at that time and again at 6 weeks after surgery. In contrast, we usually left the urinary catheter in place for 14 days and then evaluated for residual fistula via dye test immediately after catheter removal. If the dye test is negative and no other medical indications exist for hospitalization, then the patient is usually discharged from the Fistula Care Centre that day or shortly thereafter. While we do ask that our patients return for repeat evaluation for residual fistula at the Fistula Care Centre one month after surgery, only one of the two patients who had S. haematobium in our study returned for any post-operative evaluation, at 10 weeks after surgery. She did not have repeat dye testing done at that visit but was found to still have a successful fistula repair by clinical history and exam.

Given the small and cross-sectional nature of our study, we recognize several limitations. First, most women came from areas in Malawi that have a low prevalence of schistosomiasis and this could have influenced our results. Therefore, future studies that assess the relationship between obstetric VVF and schistosomiasis should comprise more women from areas that have a high prevalence of schistosomiasis. Second, although microscopy has been reported to be the most appropriate technique for detection of $S$. haematobium infection ${ }^{24} S$. haematobium host egg excretion varies throughout the day and 
this could challenge the accuracy of microscopy ${ }^{25}$. Therefore, consecutive urine specimen collection and urine microscopy for 2-3 days could improve detection of $S$. haematobium. Third, we tested for urinary schistosomiasis, not FGS, and did not sample the rectum, vagina, or bladder. Perhaps damage from an earlier chronic infection or extensive FGS is a more meaningful risk factor for VVF. Additionally, we tested only for $S$. haematobium, not $S$. mansoni, which is less common in Malawi. If S. mansoni causes a pro-inflammatory status in the bowel, it could be a risk factor for OF. Ours is the only study to evaluate the prevalence of $S$. haematobium in an obstetric fistula population in the past 45 years and the first to evaluate it in Malawi, a country with a relatively high rate of schistosomiasis infection.

\section{Conclusions}

Our findings establish that the prevalence of $S$. haematobium is low among patients in an obstetric fistula center in central Malawi and is similar to the prevalence of $S$. haematobium in the general population of Malawi. In addition, the two patients with $S$. haematobium had good surgical outcomes, and none of the six women with failed fistula repair were positive for $S$. haematobium. While there remains a plausible link between $S$. haematobium infection and poor healing after obstetric fistula surgery with subsequent failure, it was not demonstrated in this small cross-sectional study.

\section{Competing interests}

The authors declare that they have no competing interests.

\section{Acknowledgements}

Support for the use of the REDCap database was funded by the American College of Obstetricians and Gynecologists/ Kenneth Gottesfeld-Charles Hohler Memorial Foundation 2012-2013 Research Fellowship Award in Ultrasound (http:/ / www.acog.org/About-ACOG/ACOG-Departments / Research-Fellowships-andAwards/Kenneth-GottesfeldCharles-Hohler-Memorial-Foundation-Research-AwardinUltrasound). We received additional support for the use of the REDCap database from grant [1UL1TR001111] from the North Carolina Clinical and Translational Science Award program of the Division of Research Resources, National Institutes of Health. We would like to thank the Freedom From Fistula Foundation which provides care for all obstetric fistula patients at the Fistula Care Centre in Lilongwe. We also thank William Nundwe, Sandra Ngwira, and Sella Chisanga for their assistance with data collection, and Dawn Kopp and Allison Sih for assistance with data cleaning.

\section{References}

1. Vos T, Flaxman AD, Naghavi M, Lozano R, Michaud C, Ezzati M, et al. Years lived with disability (YLDs) for 1160 sequelae of 289 diseases and injuries 1990-2010: a systematic analysis for the Global Burden of Disease Study 2010. Lancet. 2012;380:2163-2196. DOI: 10.1016/ S0140-6736(12)61729-2

2. Brindley PJ, Hotez PJ. Break out: Urogenital schistosomiasis and Schistosoma haematobium infection in the post-genomic era. PLoS Negl Trop Dis [online serial]. 2013;7. Accessed at: http://www.ncbi. nlm.nih.gov/pmc/articles/PMC3610632/. Accessed March 17, 2017. DOI: 10.1371/journal.pntd.0001961

3. Steinmann P, Keiser J, Bos R, Tanner M, Utzinger J. Schistosomiasis and water resources development: Systematic review, meta-analysis, and estimates of people at risk. Lancet Infect Dis. 2006;6:411-425. DOI: $10.1016 /$ S1473-3099(06)70521-7
Africa: Review of their prevalence, distribution, and disease burden. PLoS Negl Trop Dis [online serial]. 2009;3. Accessed at: http://www. ncbi.nlm.nih.gov/pmc/articles/PMC2727001/. Accessed March 17, 2017. DOI: 10.1371/journal.pntd.0000412

5. Dennis N, Wilkinson J, Robboy S, Idrissa A. Schistosomiasis and vesicovaginal fistula. Afr J Reprod Health. 2009;13:137-140.

6. van der Werf MJ, de Vlas SJ, Brooker S, Looman CW, Nagelkerke NJ, Habbema JD, et al. Quantification of clinical morbidity associated with schistosome infection in sub-Saharan Africa. Acta Trop. 2003;86:125139. DOI: $10.1016 / \mathrm{S} 0001-706 \mathrm{X}(03) 00029-9$

7. Mbabazi PS, Andan O, Fitzgerald DW, Chitsulo L, Engels D, Downs JA. Examining the relationship between urogenital schistosomiasis and HIV infection. PLoS Negl Trop Dis. 2011;5(12): e1396. DOI: 10.1371/ journal.pntd.0001396

8. Kjetland EF, Leutscher PDC, Ndhlovu PD. A review of female genital schistosomiasis. Trends Parasitol. 2012;28:58-65. DOI: 10.1371/ journal.pntd.0001396

9. Norseth HM, Ndhlovu PD, Kleppa E, Randrianasolo BS, Jourdan PM, Roald B, et al. The colposcopic atlas of schistosomiasis in the lower female genital tract based on studies in Malawi, Zimbabwe, Madagascar and South Africa. PLoS Negl Trop Dis. 2014;8(11): e3229. DOI: 10.1371/journal.pntd.0003229

10. Hotez PJ, Fenwick A, Kjetland EF. Africa's 32 cents solution for HIV/AIDS. PLoS Negl Trop Dis. 2009;3(5): e430. DOI: 10.1371/ journal.pntd.0000430

11. Teesdale CH, Chitsulo L. Schistosomiasis in Malawi--a review. Trop Med Parasitol. 1985;36:1-6.

12. Ministry of Health and Population, Malawi. National Schistosomiasis Control Programme, Malawi: Ten year plan of action 1991-2000.

13. Bowie C, Purcell B, Shaba B, Makaula P, Perez M. A national survey of the prevalence of schistosomiasis and soil transmitted helminths in Malaŵi. BMC Infect Dis. 2004;4:49. DOI: 10.1186/1471-2334-4-49

14. Kayondo M, Wasswa S, Kabakyenga J, Mukiibi N, Senkungu J, Stenson A, et al. Predictors and outcome of surgical repair of obstetric fistula at a regional referral hospital, Mbarara, western Uganda. BMC Urol. 2011;11:23. DOI: 10.1186/1471-2490-11-23

15. Sachdev PS, Hassan N, Abbasi RM, Das CM. Genito-urinary fistula: a major morbidity in developing countries. J Ayub Med Coll Abbottabad JAMC. 2009;21:8-11.

16. Kalilani-Phiri LV, Umar E, Lazaro D, Lunguzi J, Chilungo A. Prevalence of obstetric fistula in Malawi. Int $\mathrm{J}$ Gynecol Obstet. 2010;109:204-208. DOI: 10.1016/j.ijgo.2009.12.019

17. Richter J, Lux J, Reinecke P, Müller-Mattheis V. Of flukes and fistulae. The Lancet. 2008;371:1308. DOI: 10.1016/S01406736(08)60566-8

18. Tilstra JH, Schagen Van Leeuwen JH. Impaired healing of a vaginal tear in a patient with schistosomiasis. Acta Obstet Gynecol Scand. 2001;80:770-771. DOI: 10.1034/j.1600-0412.2001.080008770.x

19. Bland KG, Gelfand M. The influence of urinary bilharziasis on vesico-vaginal fistula in relation to causation and healing. Trans $\mathrm{R}$ Soc Trop Med Hyg. 1970;64:588-592.

20. Wagatsuma Y, Aryeetey ME, Sack DA, Morrow RH, Hatz C, Kojima S. Resolution and resurgence of Schistosoma haematobiuminduced pathology after community-based chemotherapy in Ghana, as detected by ultrasound. J Infect Dis. 1999;179:1515-1522. DOI: $10.1086 / 314786$

21. Harris PA, Taylor R, Thielke R, Payne J, Gonzalez N, Conde JG. Research Electronic Data Capture (REDCap) - A metadata-driven methodology and workflow process for providing translational research informatics support. J Biomed Inform. 2009;42:377-381. DOI: 10.1016/j.jbi.2008.08.010 
22. Mtethiwa AHN, Nkwengulila G, Bakuza J, Sikawa D, Kazembe A. Extent of morbidity associated with schistosomiasis infection in Malawi: a review paper. Infect Dis Poverty. 2015;4(25). DOI: 10.1186 s40249-015-0053-1

23. Polan ML, Sleemi A, Bedane MM, Lozo S, Morgan MA. Obstetric Fistula. In: Debas HT, Donkor P, Gawande A, Jamison DT, Kruk ME, Mock CN, editors. Essent Surg Dis Control Priorities Third Ed Vol 1 [online]. Washington (DC): The International Bank for Reconstruction and Development / The World Bank; 2015. Accessed at: http://www. ncbi.nlm.nih.gov/books/NBK333495/. Accessed January 8, 2018.
24. Koukounari A, Webster JP, Donnelly CA, Bray BC, Naples J, Bosompem K, et al. Sensitivities and specificities of diagnostic tests and infection prevalence of Schistosoma haematobium estimated from data on adults in villages northwest of Accra in Ghana. Am J Trop Med Hyg. 2009;80:435-441.

25. Doehring E, Feldmeier H, Daffalla AA. Day-to-day variation and circadian rhythm of egg excretion in urinary schistosomiasis in the Sudan. Ann Trop Med Parasitol. 1983;77:587-594. 